\title{
mtDNA synthesis ignites the inflammasome
}

The NLRP3 inflammasome lies at the heart of inflammation in many immunological settings, but the exact mechanisms controlling its activation have remained elusive. Michael Karin and colleagues now report that the new synthesis of mitochondrial DNA (mtDNA) couples the priming and activation stages of NLRP3 inflammasome activation by enabling the generation of oxidized mtDNA (ox-mtDNA), which the authors suggest may be the 'ultimate' ligand for NLRP3.

Activation of the NLRP3

inflammasome requires a priming step followed by an activation step. Priming is mediated by pathogen and damage-associated molecular patterns or by inflammatory cytokines and leads to the upregulation of inflammasome components. The activation step drives NLRP3 inflammasome assembly and caspase 1 activation, but it is less clear how this occurs. As diverse stimuli can trigger the activation step, it has been proposed that a common intracellular mediator resulting from mitochondrial damage - potentially ox-mtDNA - is involved. However, mitochondrial damage in itself does not activate NLRP3 in the absence of priming, so it is unclear how these steps are linked.

Zhong et al. generated mice specifically lacking TFAM - a

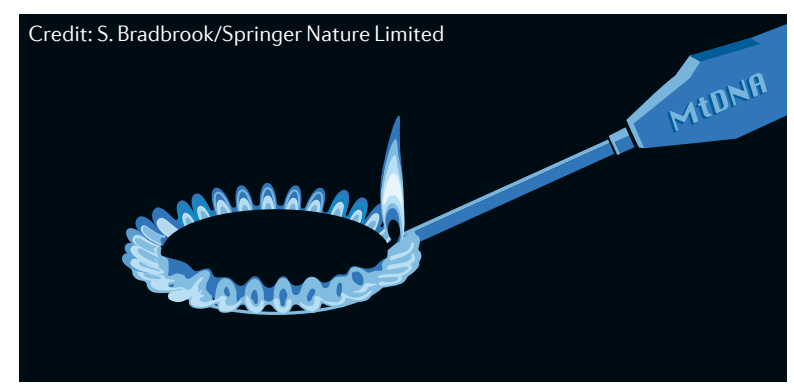

transcription factor that regulates the compaction and replication of mtDNA - in myeloid cells. Bone marrow-derived macrophages (BMDMs) from these $\mathrm{Tfam}^{\text {Mye }}$ mice had markedly reduced mtDNA levels and did not produce mitochondrial reactive oxygen species (mtROS) and ox-mtDNA in response to NLRP3 activators. They also showed defective caspase 1 activation and IL- $1 \beta$ processing in response to NLRP3 activators, despite showing normal induction of pro-IL- $1 \beta$ and other inflammasome components.

Treatment with hydrogen peroxide (the predominant ROS found in activated macrophages) induced IL- $1 \beta$ release in control BMDMs, but not in Tfam ${ }^{\text {Mye }}$ BMDMs, suggesting that mtROS activate NLRP3 by inducing ox-mtDNA. In support of this, transfection of $\mathrm{Tfam}^{\text {Mye }}$ BMDMs with synthetic mtDNA containing oxidized 8-OH-dGTP induced IL- $1 \beta$ in the absence of inflammasome activators, whereas synthetic mtDNA containing dGTP did not have this effect. Importantly, the sequence of the transfected DNA did not matter and various synthetic mtDNAs induced IL- $1 \beta$ as long as they contained 8-OH-dGTP.

To understand how ox-mtDNA is produced during priming, the authors examined mtDNA metabolism in lipopolysaccharide (LPS)-treated BMDMs. They found that LPS induced a marked increase in mtDNA synthesis in mitochondria that was dependent on the TLR signalling adaptor MYD88 at early time points, on TRIF at later time points and on IRF1 downstream of both adaptors. The authors therefore examined the IRF1 transcriptome for a target gene involved in mtDNA replication; they identified the mitochondrial deoxyribonucleotide kinase UMP-CMPK2 (CMPK2) as the rate-limiting enzyme controlling dNTP supply for LPS-induced mtDNA synthesis. Accordingly, CMPK2-deficient BMDMs did not upregulate mtDNA synthesis following LPS treatment and showed deficient caspase 1 activation and IL- $1 \beta$ release. Moreover, expression of wild-type CMPK2 in IRF-deficient BMDMs restored LPS-stimulated mtDNA replication, whereas a catalytically inactive CMPK2 did not.

Imaging experiments using labelled mtDNA confirmed that newly synthesized mtDNA colocalizes with ASC specks (which form during inflammasome assembly) in LPS-primed BMDMs treated with NLRP3 activators to induce mitochondrial damage.

Finally, the authors found that peritoneal macrophages isolated from LPS-treated IRF1-deficient mice had reduced mtDNA levels. Furthermore, IRF1-deficient mice did not show IL- $1 \beta$ production in response to LPS or alum treatment, suggesting that IRF1-mediated induction of mtDNA synthesis also regulates NLRP3 activation in vivo.

A role for ROS and mitochondria in NLRP3 activation was first proposed by Jurg Tschopp and colleagues, but became somewhat controversial. The study by Karin and colleagues sheds light on how mitochondria link the priming and activation stages during NLRP3 triggering. The authors suggest that targeting CMPK2 could offer a novel therapeutic approach in NLRP3-dependent disease settings.

Yvonne Bordon

ORIGINAL ARTICLE Zhong, Z. et al. New mitochondrial DNA synthesis enables NLRP3 inflammasome activation. Nature https://doi.org/ 10.1038/s41586-018-0372-z (2018) FURTHER READING Tschopp, J. \& Schroder, K. NLRP3 inflammasome activation: the convergence of multiple signalling pathways on ROS production? Nat. Rev. Immunol. 10, 210-215 (2010) 